\title{
Contribution des images radar ERS à la modélisation hydrologique du Coët-Dan par TopModel
}

\author{
Using radar ERS images for the hydrological modelling of \\ Coët-Dan with TopModel
}

par P. Gineste

Ecole inter-Etats d'Ingénieurs de l'Equipement Rural (E.I.E.R.)

Distributed models generally result in multiple acceptable parametrisations with respect to the fit of the simulations to the discharge, yet each parametrisation implies a different internal hydrological behaviour. Hence, new data are required to further constrain and validate distributed models.

Since these last thirty years have revealed the major role of the saturated areas dynamics under the humid and temperate climates, the saturated area extension appears as an essential information with this respect, and the radar imagery the most promising technology to fumish the expected spatialised data.

This paper presents the main results of a study of the French Coët-Dan catchment (Gineste, 1998), which aims to extract from ERS radar images an objective distributed measure of an internal state variable of the catchment (i.e. the saturated area extent), in order to validate and eventually improve TopModel simulations.

\section{INTRODUCTION}

La seconde moitié du XXe siècle a vu des avancées considérables dans la compréhension des processus du ruissellement, mettant en lumière le rôle fondamental de la dynamique des zones saturées dans la genèse des débits sous les climats humides et tempérés. Aussi, grâce à la capacité croissante des moyens informatiques, la modélisation hydrologique a connu un formidable essor : du global au distribué.

Cependant, les chemins de l'eau, depuis l'instant où une goutte de pluie rencontre la surface de la Terre jusqu'à son éventuel écoulement en rivière, dépendent d'une multitude de facteurs, eux-mêmes variables dans l'espace et dans le temps [1]. D'où la difficulté à représenter fidèlement les processus du ruissellement à partir des mesures et des méthodes conventionnelles qui trop souvent supposent l'uniformité et la stationnarité des phénomènes.

De plus, il nous faut considérer que la nature des sorties que l'on sollicite - ou plutôt que l'on voudrait solliciter - des modèles a considérablement évolué. A l'ère de la construction des barrages succède l'ère d'une gestion durable et intégrée des eaux et des sols, et qui nécessite une information spatialisée : chemins de l'eau pour le calcul des transports géochimiques et sédimentaires, flux de matière et d'énergie à l'interface sol-végétation-atmosphère, conséquences d'un changement d'occupation du sol, de changements climatiques...

Mais le problème d'une approche qui se voudrait causale et par conséquent explicative, est qu'elle aboutit vite à une situation inextricable dès lors qu'elle s'applique à un petit bassin versant réel [2]. D'ailleurs même les modèles physiquement distribués ne sont dans l'absolu que d'extrêmes simplifications de la réalité. Et rien, théoriquement, ne justifie que l'on puisse appliquer ces équations dites physiques à l'échelle de la maille d'un modèle distribué. Ce faisant néanmoins, leurs paramètres deviennent effectifs ; c'est-à-dire que, comme tout paramètre d'un modèle conceptuel, ils ne sont plus vraiment mesurables : ils perdent leur sens physique dans le changement d'échelle qui s'opère, de l'échelle de la mesure à celle de la maille. C'est pourquoi nous ne disposons à l'heure actuelle que de modèles de simulation, qui fonctionnent par un apprentissage sur les débits observés, censé permettre d'identifier la valeur effective des paramètres du modèle.

Malheureusement, il apparaît une sorte de décalage entre la quantité d'information nécessaire à la calibration d'un modèle distribué et l'information contenue dans les seules chroniques des pluies et des débits. La spatialisation 
engendre un tel degré de liberté que la connaissance du débit à l'exutoire d'un bassin ne suffit pas à contraindre efficacement le modèle, à permettre autrement dit de le paramétrer sans ambiguïté. D'où une certaine indétermination sur les flux et variables internes.

C'est encore un problème de changement d'échelle qui se pose. Non pas dans le sens des démarches agrégatives, mais parce que cela revient à utiliser une information collectée à une échelle intégrée (e.g. débit) pour effectuer des estimations à des échelles plus fines... C'est ce qu'on appelle - pas seulement en hydrologie - un problème d'équifinalité [3]. L'équifinalité marque la limite des possibilités d'inversion des paramètres fins d'un modèle à partir de mesures au niveau le plus grossier.

Ainsi, compter sur la mise en œuvre globale d'un modèle de simulation (e.g. apprentissage sur le débit à l'exutoire) pour nous renseigner sur les processus hydrologiques internes au bassin, apparaît comme un enjeu pour le moins difficile, non pas qu'une telle approche désagrégative soit impossible, mais parce qu'elle est limitée. Et la limite dépend a priori de la justesse de construction théorique du modèle, de l'hétérogénéité du bassin, et des données utilisées pour l'apprentissage du modèle [4].

Dans ce contexte, il semble bien délicat d'obtenir une simulation spatialisée susceptible d'être validée par l'observation. Pourtant c'est sûrement la meilleure réponse aux difficultés de validation des modèles distribués : valider un modèle sur la justesse de sa représentation physique interne... Faute de quoi, ces modèles fonctionnent suivant le schéma d'apprentissage et de validation des modèles globaux, i.e. comme des "boîtes noires" [5], et tout comme eux, ils ne peuvent guère alors prétendre qu'à "gonfler" les chroniques de débit..

Aussi c'est très précisément ce qui a motivé l'étude résumée dans cet article [4] : obtenir une mesure spatialisée et objective d'une variable d'état interne au bassin versant, et ce à l'aide de la télédétection radar : en chaque pixel on considère la saturation comme 0 ou 1 ; mesure susceptible d'être confrontée aux résultats de la simulation d'un modèle hydrologique, voire de les améliorer si tant est que ceux-ci ne soient pas trop incertains...

Après un bref descriptif des composantes de terrain, mathématique, et spatiale des matériels de l'étude, on présente les principaux résultats obtenus, en distinguant successivement l'objectif d'une détection des zones saturées et l'application hydrologique escomptée.

\section{II - MATÉRIELS DE L'ÉTUDE}

\section{- 2.1 Le BVRE du Coët-Dan}

Le bassin versant du Coët-Dan s'étend sur près de $12 \mathrm{~km}^{2}$ situés pour l'essentiel sur la commune de Naizin, au nord-est du Morbihan (France). La région jouit d'un climat océanique, caractérisé par une température moyenne de $11^{\circ} \mathrm{C}$ et la douceur de ses pluies. Le Coët-Dan reçoit en moyenne $711 \mathrm{~mm}$ par an et les pluies supérieures à $20 \mathrm{~mm}$ par jour sont exceptionnelles. Son relief est assez peu marqué notamment dans la partie amont où les pentes sont en général inférieures à $5 \%$.

C'est un bassin versant instrumenté et suivi par le Cemagref depuis 1971. Il a fait l'objet de nombreuses études depuis une vingtaine d'années et est bien connu pour fonc- tionner suivant un schéma de zones saturées contributives d'aire variable [6]. Il est intégré dans le réseau européen des bassins versants expérimentaux.

\section{2.2 TOPMODEL / GLUE}

Comme Keith Beven aime à le souligner, TOPMODEL n'est pas vraiment un modèle hydrologique, mais plutôt un ensemble de concepts de modélisation qui permet d'appréhender le comportement des bassins versants d'une façon distribuée en n'impliquant qu'un minimum de paramètres, et la dynamique des zones contributives en particulier [7].

TOPMODEL découle en effet d'un ensemble d'hypothèses simplificatrices sur la physique de la zone saturée qui conduisent à la notion de points hydrologiquement semblables caractérisés par une même valeur d'indice topographique. Aussi, TOPMODEL peut-il se résumer à un modèle conceptuel de nappe aquifère qui présente cette particularité de fonctionner suivant un schéma global (i.e. par bilan) tout en conservant la possibilité d'effectuer des prédictions spatialisées (par classes d'indices). C'est ainsi que se conçoit le caractère semi-distribué du modèle, particulièrement économe d'un point de vue calculatoire, et qui par conséquent permet une évaluation rigoureuse de sa capacité prédictive via la méthode GLUE (Generalised Likelihood Uncertainty Estimation).

La méthode GLUE [8], en effet, est une approche stochastique à la calibration des modèles basée sur des simulations de Monte-Carlo. Elle permet de gérer explicitement la multiplicité des jeux de paramètres acceptables pour les conditions dans lesquelles les modèles sont généralement mis en œuvre (i.e. calibrés sur les seuls débits), de quantifier l'impact des incertitudes inévitables sur les variables d'entrées et les paramètres d'un modèle, et d'assortir ainsi les simulations du modèle d'un intervalle de confiance. Elle nécessite cependant une puissance de calcul phénoménale si bien qu'elle n'est guère actuellement envisageable concernant les approches pleinement distribuées.

Le principe de la méthode consiste à générer une multitude de paramétrisations échantillonnées entre les bornes des différents paramètres du modèle (fixées a priori de façon délibérément large), à associer à chacun de ces jeux de paramètres une mesure de vraisemblance traduisant la qualité de la simulation correspondante vis-à-vis des débits observés (typiquement le critère d'efficience de Nash), et enfin à ne conserver qu'un certain pourcentage de l'ensemble des paramétrisations initialement échantillonnées qui fournit les plus fortes efficiences (les vraisemblances relatives aux paramétrisations retenues sont ensuite normalisées).

La méthode apparaît en outre comme une méthode d'apprentissage dynamique, dans la mesure où elle s'inscrit dans une logique bayesienne qui lui permet d'ajuster la vraisemblance des diverses paramétrisations retenues lorsque davantage d'observations deviennent disponibles. De plus, comme la nature de ces observations n'est pas imposée, la méthode permet d'assimiler des informations sur l'état interne d'un bassin (e.g. zones saturées) au niveau du processus d'apprentissage du modèle.

\subsection{Les images du satellite radar ERS-1}

Travaillant dans le domaine des hyperfréquences (1$15 \mathrm{GHz}$ ), ce qui l'affranchit des perturbations atmosphériques, possédant sa propre source d'illumination, ce qui lui 
permet des acquisitions de jour comme de nuit, et sensible aux propriétés physiques du milieu ausculté (i.e. structure spatiale et permittivités diélectriques), le radar est un outil potentiellement très intéressant pour l'hydrologue. Les propriétés diélectriques de la cible sont en effet étroitement liées à l'humidité superficielle du sol, du fait de la disparité des permittivités diélectriques d'un sol sec (de l'ordre de 2 à 5) et de l'eau (environ 80).

Lancé en juillet 1991, ERS-1 est le premier satellite radar permettant des acquisitions régulières de façon opérationnelle. Cette technologie sophistiquée (synthèse d'ouverture), qui procure une résolution spatiale voisine de celle des capteurs satellitaires optiques (pixel de 12,5 m), connaît depuis un développement considérable (JERS-1, ERS-2, Radarsat...).

Dans cet article, les images qui servent à la construction de l'indice présenté ci-après découlent toutes d'une des phases d'acquisition à haute répétitivité du satellite ERS-1 offrant en principe une image tous les trois jours. Ce jeu comprend effectivement 15 images PRI (PRecision Image) couvrant la période du 28 janvier au 28 mars 1992. Les divers prétraitements, notamment le filtrage, sont exposés par ailleurs [4],[9].

\section{III — DÉTECTION DES ZONES SATURÉES}

\subsection{Résultats liminaires}

Les satellites radar actuels (i.e. une seule fréquence et polarisation) ne sont pas capables de livrer une mesure précise de l'humidité des sols, y compris dans le cas favorable d'un couvert de blé homogène d'une dizaine de centimètres seulement [4] : comment déconvoluer en effet les composantes diélectrique et de rugosité de la cible à partir d'un signal unique ? Il ressort également de nos expérimentations, qu'une détection des zones saturées depuis l'espace, qui s'appuierait sur une chute du signal liée à un effet de réflexion spéculaire [10], n'est pas non plus possible.

Néanmoins, nous avons montré comment un suivi multitemporel permet, sur la période considérée, d'apprécier les variations relatives de l'humidité des sols des différents pixels d'un bassin versant, obtenant ainsi de précieuses indications sur la structure spatiale de zones qui sont homogènes en termes de dynamique de ressuyage, et qui peuvent par conséquent jouer un rôle dans la dynamique du transfert des pollutions... Nous avons également obtenu une certaine confirmation du concept de zones contributives préférentiellement localisées aux abords du ruisseau, en mettant en évidence l'influence de la dénivelée par rapport au ruisseau sur l'évolution temporelle du signal ERS [4].

\subsection{Définition d'un indice radar de saturation potentielle}

Ces résultats liminaires nous ont conduit à proposer un indice radar de saturation potentielle, défini par l'écart-type des variations temporelles du coefficient de rétrodiffusion $\sigma^{\circ}$ (Fig. 1). Cet indice est une mesure statistique des variations des propriétés diélectriques (et géométriques) des surfaces visées, et par conséquent une mesure des variations relatives de l'humidité des sols du Coët-Dan, vue la quasi-absence de couvert végétal et de travaux culturaux sur la période hivernale considérée [11].

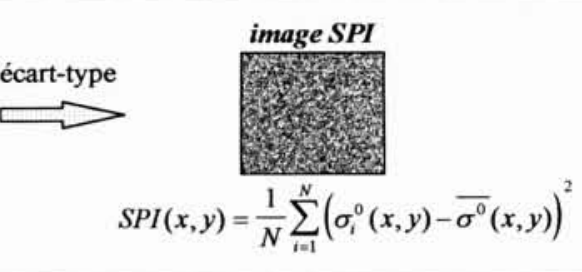

1. Calcul de l'indice de saturation potentielle SPI (Saturation Potential Index).

Le principe de détection des zones saturées est en fait ici basé sur la persistance de l'humidité des sols qui caractérise ces zones. C'est-à-dire qu'on postule que la propension des sols à se saturer est d'autant plus forte que les variations d'humidité observées y sont moindres (faible valeur d'indice). Cela suppose bien entendu de calculer l'indice SPI à partir d'une chronique d'images qui contient un épisode pluvieux marqué (suivi dans l'idéal d'une longue période de ressuyage).

Tout comme l'indice topographique de TOPMODEL, l'indice radar fournit une indication sur la propension relative des différentes zones du bassin à se saturer en surface. L'indice topographique est en fait la traduction conceptuelle d'une profondeur de nappe, qui entre autres hypothèses fait celle de l'uniformité des sols, alors que l'indice radar est une mesure statistique de la variabilité temporelle de l'humidité superficielle des sols. Le premier fournit une indication générale qui ne dépend que de la topographie du bassin alors que le second fournit une indication spécifique correspondant à la période couverte par la séquence des images radar.

Chacun apporte une information complémentaire sur la façon dont les zones saturées se développent dans l'espace d'un petit bassin versant humide, car l'indice radar est en effet susceptible d'intégrer divers facteurs non topographiques qui modifient la structure spatiale de la saturation (transmissivité latérale variable, fossés, drainage, semelle de labour...). Du point de vue de la reproduction des observations effectuées sur le sous-bassin du Mercy (1/10ème de la superficie du CoëtDan), les performances des indices topographique et radar sont tout à fait semblables, quoique relativement médiocres notamment lorsque la saturation observée est peu importante : $61 \%$ des pixels saturés le 15 février 1992 sont effectivement prédits comme tels par seuillage sur l'un ou l'autre des indices topographique et radar (de façon à reproduire la superficie effectivement saturée sur le terrain du Mercy).

\section{- 3.3. Couplage des indices topographique et radar}

On esquisse à présent deux méthodes combinant les indices topographique et radar, et qui répondent à des objectifs hydrologiques bien distincts.

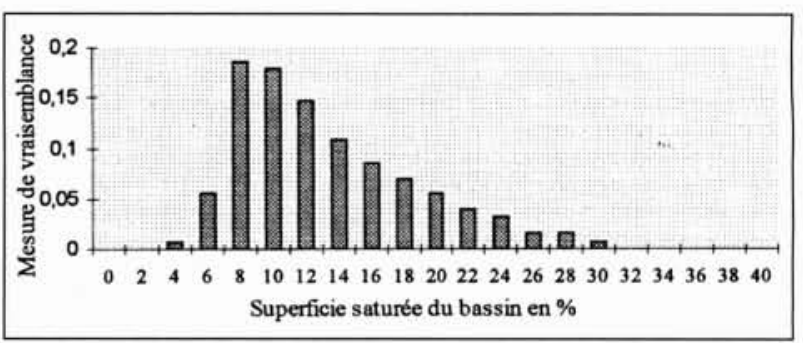

2. Estimation de la superficie globalement saturée du Coët-Dan au 15 février 1992. 


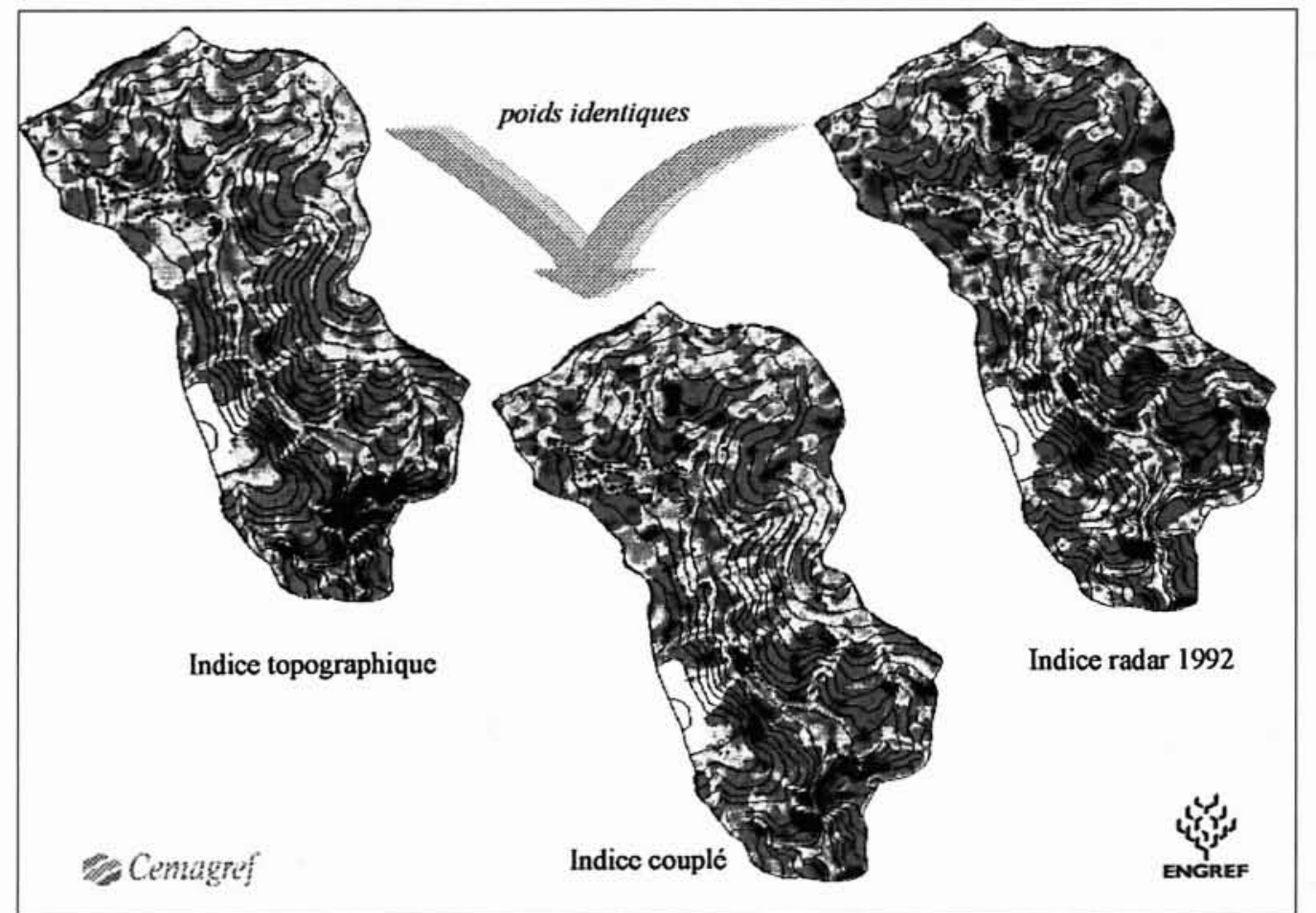

3. Distribution des valeurs de chacun des indices topographique, radar, et couplé ; étalement

dynamique par équipopulation du bleu au rouge (fort potentiel de saturation).

Les étapes du couplage sont les suivantes : calcul des moyennes et écarts-types de la distribution des valeurs de chacun des indices sur l'ensemble du bassin versant, définition commune d'une propension à la saturation normalisée fonction des écarts locaux à la moyenne spatiale, et moyenne de ces propensions normalisées.

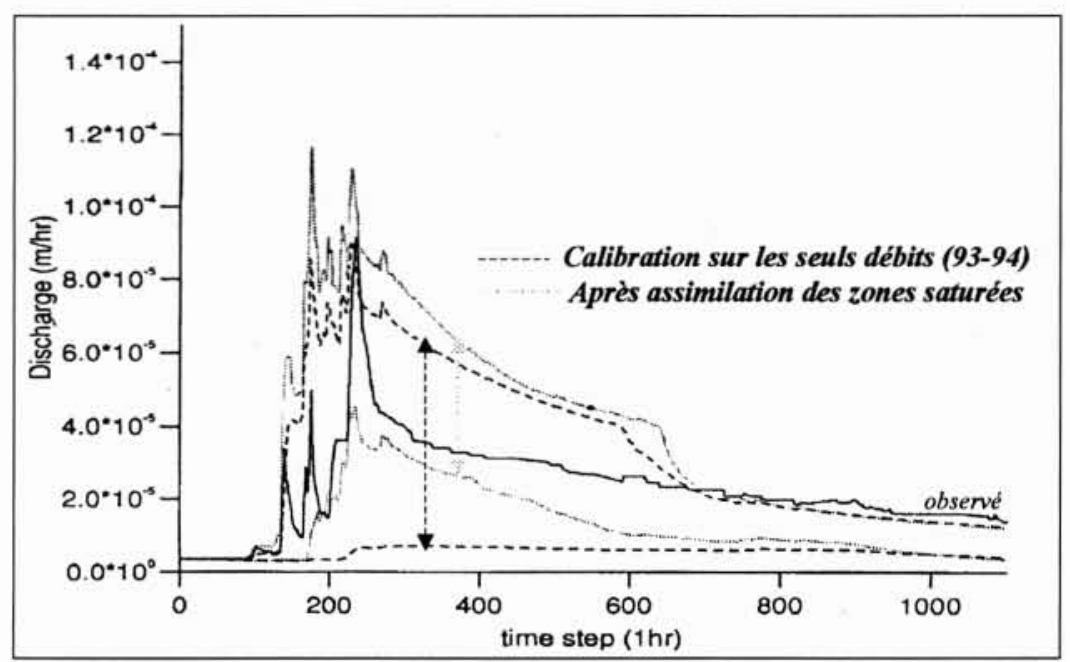

4. Intervalles de confiance à $95 \%$ sur la prédiction des débits en hiver 1992, avant et après poursuite de l'apprentissage de TOPMODEL.

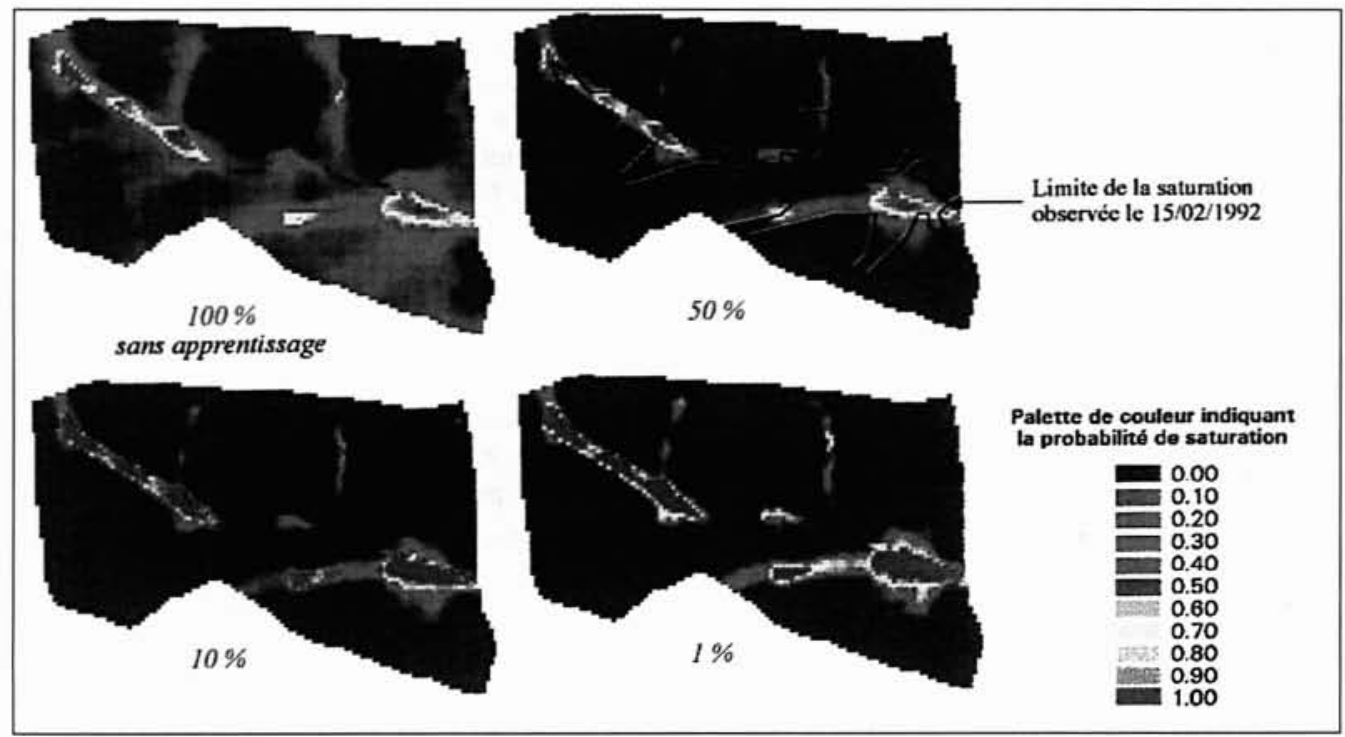

5. Simulations de l'extension des zones saturées par TOPMODEL (Zone du Mercy, $15 / 02 / 1992)$ pour divers degrés d'apprentissage sur les débits (pourcentage des paramétrisations retenues parmi les 10000 paramétrisations

initialement échantillonnées). 
La première consiste à extrapoler des observations de terrain de l'extension des zones saturées à de plus grandes superficies en s'appuyant sur un ensemble de combinaisons des indices topographique et radar, i.e. de couples de seuils sur chacun des indices. C'est-à-dire qu'inspiré par la méthode GLUE, on ne recherche pas le couple de seuils qui offre le meilleur ajustement aux observations, mais que l'on considère au contraire une multitude de couples de seuils acceptables, caractérisés chacun par une mesure de vraisemblance fonction de la qualité de l'ajustement à la saturation observée sur la zone du Mercy [12]. Alors qu'un couple de seuil unique fournirait une carte déterministe de la saturation du bassin faussement précise, cette approche vise au contraire à apprécier les incertitudes liées à l'extrapolation. Elle fournit en particulier une estimation stochastique de la superficie saturée du Coët-Dan au 15 février 1992, utilisée par la suite afin de poursuivre le processus d'apprentissage de TOPMODEL (Fig. 2).

La seconde vise à améliorer les prédictions de TOPMODEL en termes d'extension saturée. Elle fait tout d'abord l'hypothèse que TOPMODEL, à défaut de pouvoir prédire la localisation précise des zones saturées (régie par l'indice topographique), est capable de livrer une estimation raisonnable de la superficie globalement saturée du bassin. Et l'on montre comment la définition d'un indice qui combine a priori les informations topographique et radar peut permettre d'améliorer la performance des prédictions spatialisées du modèle [4]. Il suffit alors de projeter dans l'espace du bassin la saturation correspondant, non plus à une valeur particulière de l'indice topographique, mais à la valeur seuil sur ce nouvel indice qui offre globalement la même superficie saturée (Fig. 3). Concernant encore la reproduction des zones saturées observées sur la zone du Mercy le 15 février 1992, la performance de l'indice couplé atteint $68 \%$ contre $61 \%$ pour chacun des indices topographique et radar.

\section{IV — APPLICATION À LA MODÉLISA- TION HYDROLOGIQUE}

\section{- 4.1 Poursuite de l'apprentissage de TOPMODEL}

En général, l'apprentissage des modèles distribués, tel qu'il s'applique au seul débit à l'exutoire des bassins versants, se heurte au problème d'équifinalité. Et TOPMODEL, en dépit de sa paramétrisation minimale, n'y échappe pas... Cependant sa relative simplicité permet via la méthode GLUE une évaluation rigoureuse de sa capacité prédictive ainsi qu'une assimilation de l'information "zone saturée".

La méthode GLUE apparaît en effet comme une réponse pragmatique au problème d'équifinalité ; plutôt que de ne rechercher que la paramétrisation, qui sur une période particulière, fournit la meilleure efficience, la méthode tient compte de l'ensemble des paramétrisations acceptables, embrassant ainsi l'ensemble des comportements hydrologiques identifiés lors de l'apprentissage du modèle (qui, concernant notre application, consiste à associer à chacune des 10000 paramétrisations initialement échantillonnées dans l'espace des 6 paramètres du modèle une mesure de vraisemblance fonction de l'efficience des simulations correspondantes). L'ennui, c'est que la méthode met en évidence l'ampleur des incertitudes qui pèsent sur la prédiction des débits (cf. Fig.4), quelle que soit d'ailleurs la longueur des chroniques utilisées pour l'apprentissage du modèle...
Aussi cela justifie-t-il d'essayer de davantage contraindre la paramétrisation du modèle sur la base d'une information exogène aux débits. On propose donc de poursuivre l'apprentissage de TOPMODEL, initialement réalisé sur les seuls débits, sur la base d'une estimation stochastique de l'extension des zones saturées déduite des informations terrain, topographique, et radar [12]. L'incertitude associée à l'estimation de la superficie globalement saturée du bassin est explicitement incorporée dans le processus d'apprentissage du modèle à l'aide de mesures de vraisemblance (cf. Fig. 2), qui, par l'équation de Bayes, servent à tempérer les vraisemblances initiales liées à la simulation des débits (produit des mesures de vraisemblances puis normalisation). Par exemple, la vraisemblance finale d'une paramétrisation, qui le 15 février 1992, conduit à une superficie saturée non comprise entre 4 et $30 \%$ est nulle.

En dépit de l'incertitude portant sur l'estimation des zones saturées, cette poursuite de l'apprentissage de TOPMODEL conduit à rejeter de nombreuses paramétrisations précédemment acceptables (en subsistent 56 sur les 1000 offrant les meilleures efficiences en termes de reproduction des débits), à une réduction très importante de la plage de variation du paramètre de transmissivité moyenne du modèle $\left(\ln \mathrm{T}_{0}\right.$ varie de 0,3 à 1,6 pour une plage initiale de -1 à 5 ), et à une réduction sensible des incertitudes en prédiction (Fig. 4).

\subsection{Essai de validation}

Actuellement, même les démarches de validation qui plaident pour la rigueur [13], n'apportent aucune garantie quant à la justesse de la représentation physique interne du modèle... En fait, les efforts pour valider les simulations du fonctionnement hydrologique interne à un bassin sont rarissimes [14].

Cela vient en partie du fait que les mesures conventionnelles sont essentiellement ponctuelles. Et c'est pourquoi la télédétection radar alimente l'espoir d'une validation objective du concept de zones saturées contributives et des modélisations associées. Mais cela tient aussi du problème d'équifinalité. Car encore faut-il disposer d'outils de simulation capables de fournir des estimations raisonnables sur les flux et variables des versants... alors que se contenter d'une approche déterministe qui suppose l'identification d'une paramétrisation optimale semble désormais dénué de sens : concernant notre application, les meilleures paramétrisations fournissent une extension saturée, qui, lors du pic de crue du 15 février 1992, varie dans un rapport 5 !

Aussi nous proposons d'appliquer la méthode GLUE à la prédiction des zones saturées par TOPMODEL. C'est-à-dire que la prédiction finale résulte de l'ensemble des prédictions correspondant à chacune des paramétrisations acceptables du modèle au prorata de la mesure de vraisemblance qui y est associée. Elle dépend logiquement du degré d'apprentissage sur les débits, c'est-à-dire du pourcentage de paramétrisations retenues sur l'ensemble des 10000 paramétrisations initialement échantillonnées dans l'espace des 6 paramètres du TOPMODEL utilisé. Chaque pixel du bassin est ainsi caractérisé par une probabilité de saturation (espérance mathématique), qui est la somme des vraisemblances associées aux paramétrisations retenues qui prédisent la saturation en cet endroit.

Le résultat apparaît sur la figure 5 concernant la zone du Mercy et le pic de crue du 15 février 1992. L'extension des zones saturées prédite après calibration du modèle paraît relativement bien délimitée spatialement : plus de $80 \%$ de la superficie du Mercy observe une probabilité de saturation nulle ou unité contre $40 \%$ précédemment (lorsque l'estima- 
tion résulte uniquement de la plage de variation assignée à chacun des paramètres du modèle). Et cette extension crô̂t sensiblement à mesure que le critère de sélection des paramétrisations acceptables devient plus restrictif, autrement dit que le pourcentage de paramétrisations retenues diminue.

Ainsi, l'apprentissage de TOPMODEL sur la seule base des débits nous a permis d'obtenir des prédictions suffisamment bien délimitées dans l'espace du Mercy pour les confronter à la saturation effectivement observée. Cependant, elles n'atteignent dans le meilleur des cas que $41 \%$ de la superficie saturée observée, ce qui fait qu'il n'y a guère intérêt ici à les corriger à l'aide de l'indice couplé (cf. [4]). Mais il faut sans doute y voir le fait que la version du modèle utilisée suppose une transmissivité latérale des sols uniforme, alors que la transmissivité généralement bien moindre des zones de basfonds favorise une extension plus rapide de la saturation...

Se pose en effet le problème de savoir ce qu'il est réaliste d'escompter d'une confrontation avec les prédictions spatialisées d'un modèle hydrologique. Qu'est-ce qu'une prédiction adéquate lorsque le modèle n'est dans le meilleur des cas qu'une grossière approximation de l'hétérogénéité spatiale d'un bassin ? Plus de précision requiert certainement d'intégrer une transmissivité latérale variable dans le calcul de l'indice de TOPMODEL... Mais comment en pratique ?

\section{$\mathrm{V}$ - CONCLUSION}

Ce travail vise un rapprochement entre terrain et modélisation. Et nous avons choisi d'explorer la piste de la télédétection radar en essayant d'apporter une information utile à la calibration / validation des modèles qui simulent la dynamique des zones saturées.

La méthode GLUE nous a permis, d'une part de poursuivre le processus d'apprentissage de TOPMODEL sur la base d'une estimation de la superficie saturée du bassin déduite des informations terrain, topographique, et radar, d'autre part de fournir une estimation raisonnable (stochastique) de l'extension spatiale des zones saturées, offrant alors la possibilité d'une validation du modèle sur la justesse de sa représentation interne. Elle permet d'analyser les impacts respectifs de l'information "zone saturée " sur la prédiction des débits et de l'information " débit" sur la prédiction des zones saturées, soit de faire porter l'analyse tant sur la précision des estimations de débit que sur la qualité des prédictions spatialisées de TOPMODEL. Et le problème d'équifinalité semble se manifester avec plus ou moins d'acuité selon le cas considéré. C'est-à-dire que la multiplicité des paramétrisations acceptables, si elle proscrit toute approche déterministe et induit de fortes incertitudes sur la prédiction des débits, néanmoins, ne se traduit pas par des prédictions spatialisées incertaines concernant notre application. TOPMODEL semble donc échapper au problème d'une trop forte indétermination sur les processus internes qu'impliquerait un apprentissage sur les seuls débits à l'exutoire..

Cependant, chacun des objectifs de l'étude n'est que partiellement atteint. L'indice radar fournit une indication sur la propension relative des différentes zones d'un bassin à se saturer mais ne permet pas une mesure de l'extension saturée correspondant à une image particulière... Il est néanmoins remarquable que l'information obtenue déjà permette, grâce aux techniques de couplage proposées, une amélioration sensible des prédictions du modèle. Aussi l'avènement prochain de satellites radar multi-configurés, susceptibles de fournir une mesure de l'humidité superficielle des sols [15], semble offrir d'immenses perspectives en matière d'appren- tissage et de validation des modèles distribués (calibration de la distribution spatiale des valeurs de l'indice de TOPMODEL...). Et la télédétection d'apparaître comme un outil indispensable, complémentaire à la récente spatialisation des modèles, qui permettrait de mieux appréhender les conditions initiales et aux limites. Elle offre un regard nouveau sur les processus hydrologiques de surface, et est susceptible d'intervenir en amont comme en aval de la modélisation, pour aider à paramétriser le milieu (segmentation de l'espace en zones homogènes), et comme nous l'avons montré, pour limiter les incertitudes sur la paramétrisation du modèle et par conséquent les incertitudes en prédiction.

\section{VI — REMERCIEMENTS}

Ce travail a été réalisé au Laboratoire Commun de Télédétection Cemagref-ENGREF à Montpellier, au Laboratoire de Sciences du sol de l'INRA Rennes, et à l'Institut des Sciences naturelles et de l'environnement de l'Université de Lancaster en Grande-Bretagne. Que tous ceux qui y ont contribué trouvent ici l'expression de mon meilleur souvenir ainsi que la marque de toute ma gratitude.

\section{BIBLIOGRAPHIE}

[1] Ambroise, B., Genèse des débits dans les petits bassins versants ruraux en milieu tempéré : 1-Processus et facteurs, Rev. Sci. Eau, vol 4, 471495, 1998.

[2] Dooge, J.C.I., Looking for hydrological laws, Water Resour. Res., vol 22(9), 46s-58s, 1986.

[3] Beven, K., Prophecy, reality and uncertainty in distributed hydrological modelling. Adv. in Water Resour., vol 16, 41-51, 1993.

[4] Gineste, P., Contribution de l'imagerie satellitale radar ERS à la détection des zones saturées et à la modélisation d'un petit bassin versant agricole par TOPMODEL. Thèse de Doctorat de l'ENGREF, 224 pp, 1998.

[5] Marsily, G. de, Quelques réflexions sur l'utilisation des modèles en hydrologie, Rev. Sci. Eau, vol 7, 219-234, 1994.

[6] Gascuel-Odoux, C., P. Mérot, A. Crave, P. Gineste, A. Taha, et Z. Zhang, Les zones contributives de fond de vallée : localisation, structure et fonctionnement hydrodynamique, in : Le programme CORMORAN Caractérisation, ObseRvation, Modélisation des tRansferts en milieu Agricole iNtensif, Ed. C. Cheverry, INRA Editions, 129-142, 1996.

[7] Beven, K.J., R. Lamb, P.F. Quinn, R. Romanowicz, et J. Freer. TOPMODEL. in : Computer Models of Watershed Hydrology, V.P. Singh (Ed.), Water Resour. Publ., Highlands Ranch, Colorado, 1995.

[8] Beven, K., and A. Binley, The future of distributed models : Model calibration and uncertainty prediction, Hydrol. Process., vol 6, 279-298, 1992

[9] Gineste, P., A simple, efficient filter for multi-temporal SAR images, International Journal of Remote Sensing, vol 20(13), 2565-2576, 1999.

[10] Brun, C., R. Bernard, D. Vidal-Madjar, C. Gascuel-Odoux, P. Mérot, J. Duchesne, and $\mathrm{H}$. Nicolas, Mapping saturated areas with a helicopterborne C band scatterometer. Water Resour. Res., vol 26, 945-955, 1990.

[1I] Gineste, P., C. Puech, and P, Mérot, Radar remote sensing of the source areas from the Coët-Dan catchment, Hydrol. Process, vol 12, 267-284, 1998.

[12] Franks, S., P. Gineste, K. Beven, and P. Mérot, On constraining the predictions of distributed models : The incorporation of fuzzy estimates of saturated areas into the calibration process, Water Resour. Res., vol 34 $\mathrm{n}^{\circ} 4,787-797,1998$

[13] Ewen, J., and G. Parkin, Validation of catchment models for predicting land-use and climate change impacts. 1. Method., J. Hydrol., vol 175 , 583-594, 1996.

[14] Ambroise, B., J.L. Perrein, and D. Reutenauer, Multicriterion validation of a semidistributed conceptual modeling of the water cycle in the Fech catchment (Vosges Mountains, France), Water Resour. Res., vol 31. 1467-1481, 1995,

[15] Ulaby, F.T., P.C. Dubois, and J. Van Zyl, Radar mapping of surface soil moisture, J. Hydrol., vol 184, 57-84, 1996. 